\title{
Search for additional neutral Higgs bosons in the MSSM
}

\author{
Junichi Tanaka*, on the behalf of the ATLAS Collaboration \\ International Center of Elementary Particle Physics, The University of Tokyo \\ E-mail: jtanaka@icepp.s.u-tokyo.ac.jp
}

Two searches for neutral heavy Higgs: $H / A \rightarrow \tau \tau$ and $A \rightarrow Z h, h \rightarrow b \bar{b}$ are presented with $36.1 \mathrm{fb}^{-1}$ of data at $\sqrt{s}=13 \mathrm{TeV}$ recorded by the ATLAS detector at the LHC. There is no statistically significant excess. Upper limits on cross-section times branching fraction and the interpretation of results in the $m_{A}-\tan \beta$ plane are shown within the MSSM. Large areas of high $\tan \beta$ are excluded by $H / A \rightarrow \tau \tau: \tan \beta>15$ for $m_{A}=1 \mathrm{TeV}$ in the hMSSM scenario at the $95 \%$ confidence level.

The 39th International Conference on High Energy Physics (ICHEP2018)

4-11 July, 2018

Seoul, Korea

* Speaker. 


\section{Introduction}

We have learned several things so far from LHC results: There is one Higgs boson with $m_{h}=125 \mathrm{GeV}$, spin 0 and $C P$-even [1]. Its couplings to bosons and fermions are consistent with the Standard Model (SM) prediction. There is no SUSY up to $\sim 2 \mathrm{TeV}$ for gluino/sqaurk, $\sim 1 \mathrm{TeV}$ for scalar top ${ }^{1}$ [2]. There is no other exotic particles up to a few $\mathrm{TeV}$ depending on models [3]. The SM is successful but we have strong motivations to search for physics beyond the SM (BSM): Hierarchy problem, dark matter, GUT etc. Since many new physics models require two Higgs doublets (2HDM), a search for additional neutral Higgs is one of general ways for BSM searches. For high $\tan \beta$ ( $\beta$ is a ratio of two vacuum expectation values), down-type fermion channels (produced from the decay of the heavy neutral Higgs) are promising and for low $\tan \beta$, up-type fermion, $V V$ and $h h$ channels are promising in the MSSM. In this proceedings, we show two searches at ATLAS [4] with $36.1 \mathrm{fb}^{-1}$ collected at $\sqrt{s}=13 \mathrm{TeV}: H / A \rightarrow \tau \tau$ and $A \rightarrow Z h, h \rightarrow b \bar{b}$.

\section{2. $H / A \rightarrow \tau \tau$}

$b$-associated production $(b b H / A)$ cross-section becomes larger as $\tan \beta$ higher in the MSSM and also the branching fraction of $H / A \rightarrow \tau \tau$ gets larger as $\tan \beta$ gets higher. $\tau \tau$ final state with $b b H / A$ is one of the most important channels in the MSSM Higgs search. Both leptonhadron $\left(\tau_{\text {lep }} \tau_{\text {had }}\right)$ and hadron-hadron $\left(\tau_{\text {had }} \tau_{\text {had }}\right)$ channels based on the tau-lepton decay have good sensitivity in this search.

Main background events come from mis-identification of tau-lepton: multi-jets, $W$-jets etc. The contribution from $Z \rightarrow \tau \tau$ is small in the high mass region. Categories of $b$-veto and $b$-tag are introduced to enhance gluon fusion and $b b H / A$ production, respectively. The total transverse mass $m_{\mathrm{T}}^{\text {tot }}$, which is defined as $m_{\mathrm{T}}^{\text {tot }}=\sqrt{\left(p_{\mathrm{T}}^{\tau_{1}}+p_{\mathrm{T}}^{\tau_{2}}+E_{\mathrm{T}}^{\text {miss }}\right)^{2}-\left(\boldsymbol{p}_{\mathrm{T}}^{\tau_{1}}+\boldsymbol{p}_{\mathrm{T}}^{\tau_{2}}+\boldsymbol{E}_{\mathrm{T}}^{\text {miss }}\right)^{2}}$, is shown in Figs. 1 for $\tau_{\text {lep }} \tau_{\text {had }}$ with $b$-veto and $\tau_{\text {had }} \tau_{\text {had }}$ with $b$-tag. No significant excess is observed. The cross-section times branching fraction for gluon-fusion and $b b H / A$ productions is obtained and the latter is shown in Fig. 1 (right). Results from both $\tau_{\text {lep }} \tau_{\text {had }}$ and $\tau_{\text {had }} \tau_{\text {had }}$ channels are statistically combined. Upper limits are set to be up to $\sim(3-10) \mathrm{fb}$ at $m_{A}>1 \mathrm{TeV}(95 \% \mathrm{CL})$. The leptonhadron channel is sensitive below $\sim 600 \mathrm{GeV}$ but hadron-hadron becomes more sensitive above $\sim 600 \mathrm{GeV}$.

\section{3. $A \rightarrow Z h, h \rightarrow b \bar{b}$}

A search for $A \rightarrow Z h$ is sensitive in low $\tan \beta$ regions. In addition, when $b$-association in the production $(b b A)$ is considered, this channel can be enhanced in high $\tan \beta$ regions.

Lepton categories: 0, 2 charged-leptons are introduced based on the $Z$ boson decay. As a $C P$-odd Higgs boson $A$ mass gets heavier, the $125 \mathrm{GeV}$ Higgs boson $h$ is boosted. As a result, the distance between two $b$-jets from $h$ becomes closer. From this consideration, two categories are introduced: resolved and merged. In the resolved category, two $b$-jets are reconstructed with a nominal jet radius $(R=0.4)$ but in the merged category, two $b$-jets are reconstructed as a single

\footnotetext{
${ }^{1}$ The values of these limits depends on SUSY parameters.
} 

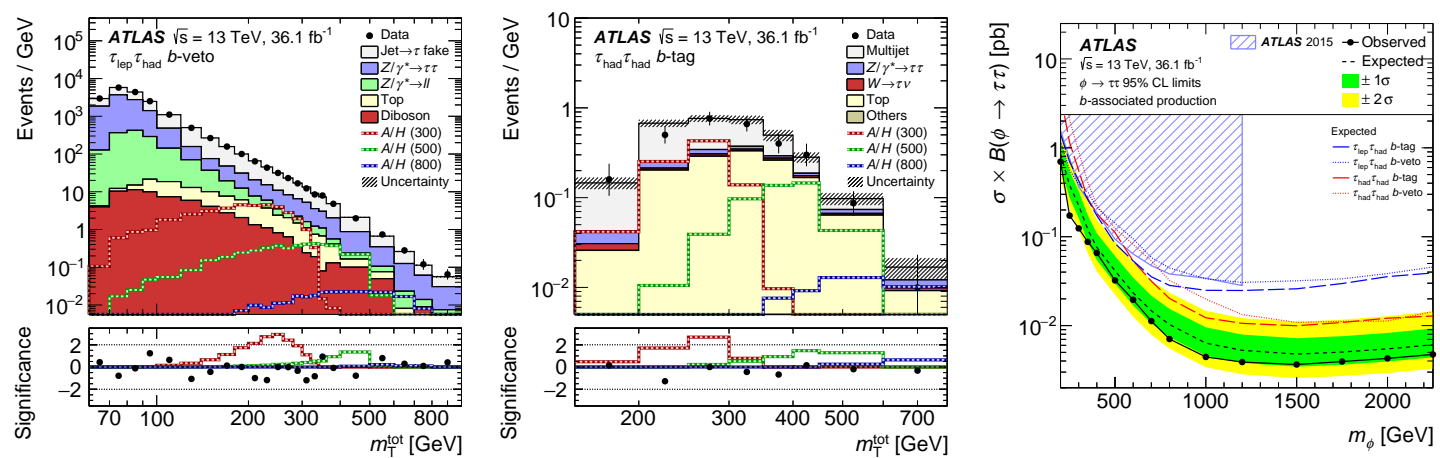

Figure 1: Distributions of $m_{\mathrm{T}}^{\text {tot }}$ for (left) $b$-veto category of $\tau_{\text {lep }} \tau_{\text {had }}$ and (middle) $b$-tag category of $\tau_{\text {had }} \tau_{\text {had }}$ channels. The combined prediction for $A$ and $H$ bosons with $300,500,800 \mathrm{GeV}$ and $\tan \beta=10$ in the hMSSM scenario are shown. (Right) the observed and expected 95\% CL upper limits on the production cross-section times branching fraction for a scalar boson via $b$-associated production. All plots are taken from Ref. [5].

jet with a large-radius $(R=1.0)$. A mass distribution of the heavy Higgs boson candidate $m_{V h}$ is shown in the left of Figs. 2 for the 2-lepton channel, where a $125 \mathrm{GeV}$ Higgs boson candidate is reconstructed as a large- $R$ jet. For the $b b A$ search, 3 or more $b$-tags is required and a transverse mass of the heavy Higgs boson candidate $m_{\mathrm{T}, V h}$ is shown in the middle of Figs. 2 for the 0 -lepton channel. In all the categories, no significant excess is observed. Upper limits on the crosssection times branching fraction for gluon-fusion and $b b A$ production are set to be $\sim(1-10) \mathrm{fb}$ at $m_{A}>1 \mathrm{TeV}(95 \% \mathrm{CL})$. Results are also interpreted in term of 2HDM Type-II, where the MSSM is one of them, as shown in the right of Figs. 2 for $m_{A}=300 \mathrm{GeV}$ and large areas except for so-called alignment limit $(\beta-\alpha=\pi / 2)$ are excluded, where $\alpha$ is a mixing angles between up-type and down-type neutral Higgs.
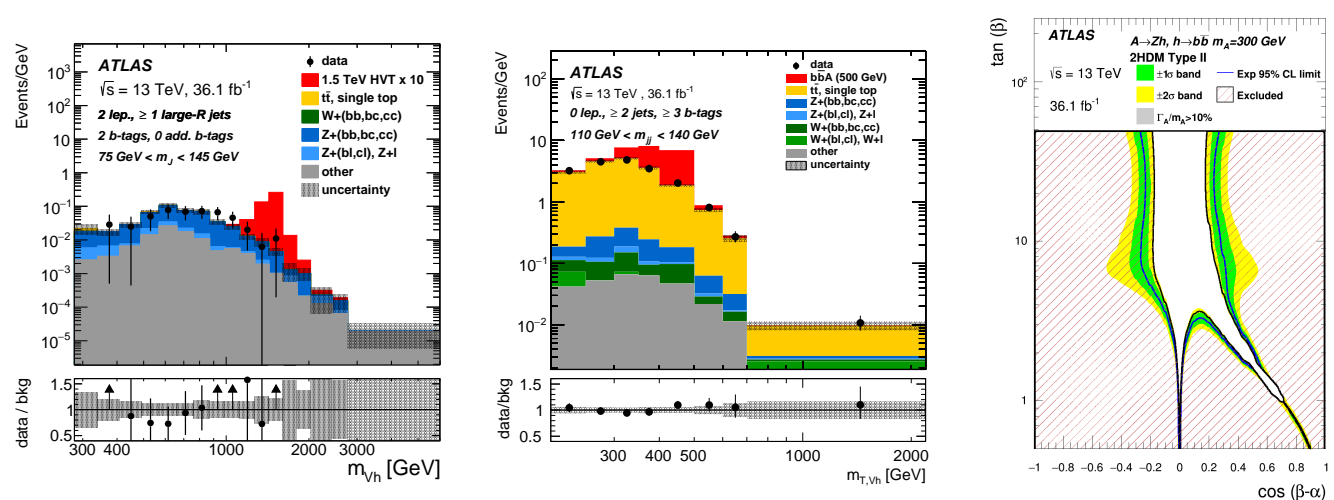

Figure 2: (Left) a $m_{V h}$ distribution corresponding to the mass of heavy neutral Higgs boson candidates for the 2-lepton channel in the merged category. The signal for the benchmark heavy vector triplet (HVT) ModelA with $1.5 \mathrm{TeV}$ normalized to 10 times the theoretical cross-section is shown by red color. (Middle) a $m_{\mathrm{T}, V h}$ distribution for the 0-lepton channel with $\geq 3 b$-jets for the $b b A$ search in the resolved category. The signal for an $A$ boson with $500 \mathrm{GeV}$ is shown by red color using a cross-section of $5 \mathrm{pb}$. (Right) the interpretation of the cross-section limits in the 2HDM Type-II in the $\cos (\beta-\alpha)$-tan $\beta$ plane for $m_{A}=$ $300 \mathrm{GeV}$. Both gluon fusion and $b b A$ productions are taken into account. All plots are taken from Ref. [6]. 


\section{Summary}

Two searches for heavy neutral Higgs: $H / A \rightarrow \tau \tau$ and $A \rightarrow Z h, h \rightarrow b \bar{b}$ with $36.1 \mathrm{fb}^{-1}$ of data collected at $\sqrt{s}=13 \mathrm{TeV}$ in the ATLAS experiment are presented. No statistically significant excess is observed. Upper limits on cross-section times branching fraction are obtained for them. Lastly Figures 3 show the interpretation of limits in the $m_{A}$-tan $\beta$ plane with several analyzes for the hMSSM scenario. Large areas of high $\tan \beta$ are excluded by $H / A \rightarrow \tau \tau$, which is displayed by gray of the left plot and also in the right plot: $\tan \beta>15$ for $m_{A}=1 \mathrm{TeV}, \tan \beta>42$ for $m_{A}=1.5 \mathrm{TeV}$ at the $95 \% \mathrm{CL}$. The result from $A \rightarrow Z h, h \rightarrow b \bar{b}$ but with the only gluon-fusion production is shown by yellow.
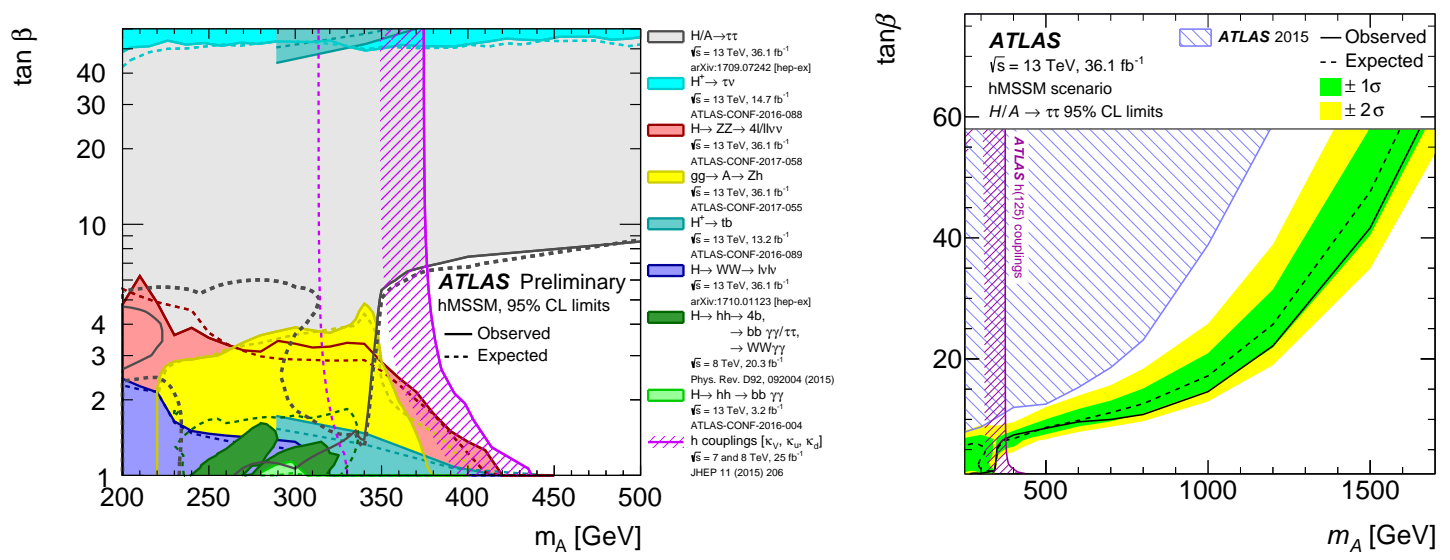

Figure 3: Regions of the $m_{A}$-tan $\beta$ plane excluded in the hMSSM model via direct searches (left) for heavy Higgs bosons and fits to the measured rates of observed Higgs boson production and decays [7] and (right) for $H / A \rightarrow \tau \tau$ [5]. Limits are quoted at 95\% CL and are indicated for the data (solid lines) and the expectation for the SM Higgs sector (dashed lines). Results of ATLAS-CONF-2017-055 shown in the plot are superseded by Ref. [6] (Section 3).

\section{References}

[1] ATLAS and CMS Collaborations, JHEP 1608 (2016) 045 [arXiv:1606.02266 [hep-ex]], ATLAS Collaboration, Eur. Phys. J. C 75 (2015) no.10, 476 [arXiv:1506.05669 [hep-ex]]

[2] ATLAS https://atlas.web.cern.ch/Atlas/GROUPS/PHYSICS/CombinedSummaryPlots/SUSY/, CMS http://cms-results.web.cern.ch/cms-results/public-results/publications/SUS/

[3] ATLAS https://atlas.web.cern.ch/Atlas/GROUPS/PHYSICS/CombinedSummaryPlots/EXOTICS/, CMS http://cms-results.web.cern.ch/cms-results/public-results/publications/EXO/

[4] ATLAS Collaboration, JINST 3 (2008) S08003

[5] ATLAS Collaboration, JHEP 1801 (2018) 055 [arXiv:1709.07242 [hep-ex]]

[6] ATLAS Collaboration, JHEP 1803 (2018) 174 [arXiv:1712.06518 [hep-ex]]

[7] ATLAS, Summary plots from the ATLAS Higgs physics group, https://atlas.web.cern.ch/Atlas/GROUPS/PHYSICS/CombinedSummaryPlots/HIGGS/ 